\title{
Effects of Coulomb Central Potential Induced by Lorentz Symmetry Breaking on Relativistic Quantum Oscillator
}

\author{
Faizuddin Ahmed ${ }^{1}$ \\ National Academy, Gauripur-783331, Assam, India
}

\begin{abstract}
In this paper, we consider the effects of a radial electric field and a constant magnetic field induced by Lorentz symmetry violation on a generalized relativistic quantum oscillator by choosing a function $f(r)=b_{1} r+\frac{b_{2}}{r}$ in the equation subject to a Cornell-type potential $S(r)=\eta_{L} r+\frac{\eta_{c}}{r}$ introduce by modifying the mass term in the equation. We show that the analytical solutions to the Klein-Gordon oscillator can be achieved, and a quantum effect is observed due to the dependence of the angular frequency of the oscillator on the quantum numbers of the system.
\end{abstract}

Keywords: Lorentz symmetry violation, Relativistic wave-equations, scalar potential, electric \& magnetic field, biconfluent Heun equation

PACS Number(s): 03.65.Pm, 11.30.Cp, 11.30.Qc

\section{Introduction}

In this paper, we investigate the behaviour of a scalar particle by solving the generalized Klein-Gordon oscillator $[1,2,3,4,5,6,7]$ in a possible scenario of anisotropy generated by a Lorentz symmetry breaking term defined by a tensor $\left(K_{F}\right)_{\mu \nu \alpha \beta}$ that governs the Lorentz symmetry violation out of the Standard Model Extension [8, 9]. The scenario of the violation of Lorentz

\footnotetext{
${ }^{1}$ faizuddinahmed15@gmail.com ; faiz4U.enter@rediffmail.com
} 
symmetry is determined by a field configuration of crossed electric and magnetic field that gives rise to a Coulomb-type Poynting vector. Then, we search for relativistic bound state solutions to the generalized KG-oscillator under the influence of a Cornell-type scalar potential in the background of Lorentz symmetry violation. In recent decades, studies of the violation of the Lorentz symmetry have been made in several branches of physics $[10,11$, $12,13,14,15,16,17,18,19,20,21,22,23,24,25,26,27,28,29,30,31]$.

Inspired by Refs. [8, 9, 32, 33, 34, 35, 36, 37, 38, 39], effects of the Lorentz symmetry violation by introducing a nonminimal coupling into the generalized KG-oscillator given by

$$
p^{\mu} p_{\mu} \rightarrow\left(p^{\mu}+i M \omega X^{\mu}\right)\left(p_{\mu}-i M \omega X_{\mu}\right)+\frac{\alpha}{4}\left(K_{F}\right)_{\mu \nu \alpha \beta} F^{\mu \nu}(x) F^{\alpha \beta}(x),
$$

where $\alpha$ is a constant, $F_{\mu \nu}(x)$ is the electromagnetic field tensor, $\left(K_{F}\right)_{\mu \nu \alpha \beta}$ corresponds to a tensor that governs the Lorentz symmetry violation out of the Standard Model Extension, $\omega$ is the oscillator frequency, and $X_{\mu}=$ $(0, f(r), 0,0)$ is a function. For Klein-Gordon oscillator we have $f(r)=r$.

\section{Generalized KG-oscillator Under the Ef- fects of Lorentz Symmetry Violation}

We consider the Minkowski flat space-time

$$
d s^{2}=-d t^{2}+d r^{2}+r^{2} d \phi^{2}+d z^{2}
$$

The generalized KG-oscillator under the effects of Lorentz symmetry violation described earlier is given by

$$
\begin{aligned}
& {\left[-\frac{\partial^{2}}{\partial t^{2}}+\frac{1}{r}\left(\frac{\partial}{\partial r}+M \omega f(r)\right)\left(r \frac{\partial}{\partial r}-M \omega r f(r)\right)+\frac{\partial^{2}}{\partial z^{2}}+\frac{1}{r^{2}} \frac{\partial^{2}}{\partial \phi^{2}}\right] \Psi} \\
& +\frac{\alpha}{4}\left(K_{F}\right)_{\mu \nu \alpha \beta} F^{\mu \nu}(x) F^{\alpha \beta}(x) \Psi=(M+S(r))^{2} \Psi
\end{aligned}
$$

where $S(r)$ is the scalar potential. 
Using the the properties of the tensor $\left(K_{F}\right)_{\mu \nu \alpha \beta}[8,9,32,33,34,35,36$, $37,38,39]$, we can rewrite (3) in the form :

$$
\begin{aligned}
& {\left[-\frac{\partial^{2}}{\partial t^{2}}+\frac{\partial^{2}}{\partial r^{2}}+\frac{1}{r} \frac{\partial}{\partial r}+\frac{1}{r^{2}} \frac{\partial^{2}}{\partial \phi^{2}}+\frac{\partial^{2}}{\partial z^{2}}-M^{2} \omega^{2} f^{2}(r)-M \omega\left(f^{\prime}(r)+\frac{f(r)}{r}\right)\right] \Psi} \\
& +\left[-\frac{\alpha}{2}\left(\kappa_{D E}\right)_{i j} E^{i} E^{j}+\frac{\alpha}{2}\left(\kappa_{H B}\right)_{j k} B^{i} B^{j}-\alpha\left(\kappa_{D B}\right)_{j k} E^{i} B^{j}\right] \Psi \\
& =(M+S(r))^{2} \Psi .
\end{aligned}
$$

Let us consider a possible scenario of Lorentz symmetry violation determined by only one non-null component of the tensor $\left(\kappa_{D B}\right)_{j k}$ as being $\left(\kappa_{D B}\right)_{13}=\kappa=$ const and by a field configuration given by $[37,38]$ :

$$
\vec{B}=B_{0} \hat{z} \quad, \quad \vec{E}=\frac{\lambda}{r} \hat{r}
$$

where $B_{0}$ is a constant, $\hat{z}$ is a unit vector in the $z$-direction and $\lambda$ is a constant associated with a linear distribution of electric charge along the axial direction. Hence Equation (4) becomes

$$
\begin{aligned}
& {\left[-\frac{\partial^{2}}{\partial t^{2}}+\frac{\partial^{2}}{\partial r^{2}}+\frac{1}{r} \frac{\partial}{\partial r}+\frac{1}{r^{2}} \frac{\partial^{2}}{\partial \phi^{2}}+\frac{\partial^{2}}{\partial z^{2}}-M^{2} \omega^{2} f^{2}(r)-M \omega\left(f^{\prime}(r)+\frac{f(r)}{r}\right)\right] \Psi} \\
& -\frac{\alpha \lambda B_{0} \kappa}{r} \Psi=(M+S(r))^{2} \Psi .
\end{aligned}
$$

Since the metric is independent of time and symmetrical by translations along the $z$-axis, as well by rotations. It is reasonable to write the solution to Eq. (6) as

$$
\Psi(t, r, \phi, z)=e^{i(-E t+l \phi+k z)} \psi(r),
$$

where $E$ is the energy of the particle, $l=0, \pm 1, \pm 2, \ldots$ are the eigenvalues of the $z$-component of the angular momentum operator, and $k$ is a constant.

Substituting the solution (7) into the Eq. (6), we obtain the following radial wave-equation for $\psi(r)$ :

$$
\begin{aligned}
& \psi^{\prime \prime}(r)+\frac{1}{r} \psi^{\prime}(r)+\left[E^{2}-k^{2}-\frac{l^{2}}{r^{2}}-M^{2} \omega^{2} f^{2}-M \omega\left(f^{\prime}+\frac{f}{r}\right)\right] \psi(r) \\
& -\frac{\alpha \lambda B_{0} \kappa}{r} \psi(r)=(M+S(r))^{2} \psi(r)
\end{aligned}
$$


In this work, we are mainly interest on Cornell-type potential which is given by $[2,3,40,41,42]$

$$
S(r)=\eta_{L} r+\frac{\eta_{c}}{r}
$$

To study the generalized KG-oscillator, we have chosen the function $f(r)=b_{1} r+\frac{b_{2}}{r}[1,2,3,4,5,6,7]$, where $b_{1}>0, b_{2}>0$ are arbitrary constants. Substituting potential (9) and using the function $f(r)$ into the Eq. (8), we obtain the following radial wave-equation :

$$
\left[\frac{d^{2}}{d r^{2}}+\frac{1}{r} \frac{d}{d r}+\Lambda-\Omega^{2} r^{2}-\frac{j^{2}}{r^{2}}-\frac{a}{r}-b r\right] \psi(r)=0,
$$

where

$$
\begin{aligned}
& \Lambda=E^{2}-M^{2}-k^{2}-2 M \omega b_{1}-2 M^{2} \omega^{2} b_{1} b_{2}-2 \eta_{L} \eta_{c} \\
& \Omega=\sqrt{M^{2} \omega^{2} b_{1}^{2}+\eta_{L}^{2}} \\
& j=\sqrt{l^{2}+M^{2} \omega^{2} b_{2}^{2}+\eta_{c}^{2}} \\
& a=\alpha \lambda B_{0} \kappa+2 M \eta_{c} \\
& b=2 M \eta_{L} .
\end{aligned}
$$

Transforming $x=\sqrt{\Omega} r$ into the Eq. (13), we obtain the following equation:

$$
\left[\frac{d^{2}}{d x^{2}}+\frac{1}{x} \frac{d}{d x}+\zeta-x^{2}-\frac{j^{2}}{x^{2}}-\frac{\eta}{x}-\theta x\right] \psi(x)=0,
$$

where

$$
\zeta=\frac{\Lambda}{\Omega} \quad, \quad \eta=\frac{a}{\sqrt{\Omega}} \quad, \quad \theta=\frac{b}{\Omega^{\frac{3}{2}}} .
$$

Now, we use the appropriate boundary conditions that the wave-functions is regular both at $x \rightarrow 0$ and $x \rightarrow \infty$. Suppose the possible solution to the Eq. (12) is

$$
\psi(x)=x^{j} e^{-\frac{1}{2}(x+\theta) x} H(x) .
$$


Substituting the solution (14) into the Eq. (12), we obtain the following equation

$$
H^{\prime \prime}(x)+\left[\frac{1+2 j}{x}-2 x-\theta\right] H^{\prime}(x)+\left[-\frac{\beta}{x}+\Theta\right] H(x)=0,
$$

where

$$
\Theta=\zeta+\frac{\theta^{2}}{4}-2(1+j) \quad, \quad \beta=\eta+\frac{\theta}{2}(1+2 j)
$$

Equation (15) is the biconfluent Heun's differential equation [40, 2, 3, 6, 7, $43,44]$ with $H(x)$ is the Heun polynomials function.

The above equation (15) can be solved by the Frobenius method. Writing the solution as a power series expansion around the origin [45]:

$$
H(x)=\sum_{i=0}^{\infty} d_{i} x^{i}
$$

Substituting the power series solution into the Eq. (15), we obtain the following recurrence relation

$$
d_{n+2}=\frac{1}{(n+2)(n+2+2 j)}\left[\{\beta+\theta(n+1)\} d_{n+1}-(\Theta-2 n) d_{n}\right] .
$$

With few coefficients are

$$
\begin{aligned}
& d_{1}=\left[\frac{\eta}{1+2 j}+\frac{\theta}{2}\right] d_{0}, \\
& d_{2}=\frac{1}{4(1+j)}\left[(\beta+\theta) d_{1}-\Theta d_{0}\right] .
\end{aligned}
$$

The power series expansion $H(x)$ becomes a polynomial of degree $n$ by imposing the following two conditions $[40,2,3,6,7]$

$$
\begin{aligned}
\Theta & =2 n, \quad(n=1,2, \ldots) \\
d_{n+1} & =0 .
\end{aligned}
$$

By analyzing the first condition, we obtain following energy eigenvalue $E_{n, l}$ :

$$
\begin{aligned}
E_{n, l}^{2} & =M^{2}+k^{2}+2 M \omega b_{1}+2 M^{2} \omega^{2} b_{1} b_{2}+2 \eta_{L} \eta_{c}+2 \Omega\left(n+1+\sqrt{l^{2}+M^{2} \omega^{2} b_{2}^{2}+\eta_{c}^{2}}\right) \\
& -\frac{M^{2} \eta_{L}^{2}}{\Omega^{2}} .
\end{aligned}
$$


Note that Eq. (21) is not the general expression of the relativistic energy eigenvalues of a generalized KG-oscillator. One can obtain the individual energy levels $E_{1, l}, E_{2, l}, E_{3, l}, \ldots$ and wave-function $\Psi_{1, l}, \Psi_{2, l}, \ldots$ one by one by imposing the additional recurrence condition $c_{n+1}=0$ on the eigenvalue problem. It should be noted here that for $b_{2} \rightarrow 0, \eta_{c} \rightarrow 0$, the energy eigenvalues (21) reduces to the result obtained in [38] (see Eq. (32) in the Ref. [38]). Thus we can see that the presence of an extra Coulomb-like scalar potential and the generalized function $f(r)$ modifies the energy spectrum of a generalized KG-oscillator field and shifted the energy levels.

The corresponding wave-functions are given by

$$
\psi_{n, l}(x)=x^{\sqrt{l^{2}+M^{2} \omega^{2} b_{2}^{2}+\eta_{c}^{2}}} e^{-\frac{1}{2}\left[x+\frac{2 M \eta_{L}}{\Omega^{\frac{3}{2}}}\right] x} H(x) .
$$

Now, we evaluate the individual energy levels and eigenfunctions one by one as in $[40,2,3,6,7]$. For example, $n=1$, we have $\Theta=2$ and $c_{2}=0$ which implies

$$
\begin{aligned}
& \Rightarrow \frac{2}{\beta+\theta} d_{0}=\left(\frac{\eta}{1+2 j}+\frac{\theta}{2}\right) d_{0} \\
& \Rightarrow \Omega_{1, l}^{3}-\left[\frac{a^{2}}{4\left(\frac{1}{2}+j\right)}\right] \Omega_{1, l}^{2}-\left[\frac{M a \eta_{L}(1+j)}{\left(\frac{1}{2}+j\right)}\right] \Omega_{1, l}-\left(\frac{3}{2}+j\right) M^{2} \eta_{L}^{2}=0
\end{aligned}
$$

a constraint on the parameter $\Omega_{1, l}$ that is on the angular frequency of the oscillator. Note that its values changes for each quantum number $n$ and $l$, so we have labeled $\Omega \rightarrow \Omega_{n, l}$. This third degree algebraic equation has at least one real root and it is exactly this real solution that gives a first degree polynomial to the function $H(x)$.

The angular frequency of the oscillator for the radial mode $n=1$ is

$$
\omega_{1, l}=\frac{1}{M b_{1}} \sqrt{\Omega_{1, l}^{2}-\eta_{L}^{2}}
$$

We can see, Eq. (24) gives us the possible values of the oscillator frequency for the radial mode $n=1$ and it depends on the quantum numbers $\{n, l\}$ 
of the system, and the Lorentz symmetry breaking parameters $\left(\alpha, B_{0}, \lambda, \kappa\right)$. Further, for each relativistic energy level and wave-function, we have a different relation of this angular frequency of the oscillator with these parameters.

The ground state energy level for the radial mode $n=1$ is given by

$$
\begin{aligned}
E_{1, l}^{2}= & M^{2}+k^{2}+2 M \omega_{1, l} b_{1}+2 M^{2} \omega_{1, l}^{2} b_{1} b_{2}+2 \eta_{L} \eta_{c} \\
& +2 \Omega_{1, l}\left(2+\sqrt{l^{2}+M^{2} \omega_{1, l}^{2} b_{2}^{2}+\eta_{c}^{2}}\right)-\left(\frac{M \eta_{L}}{\Omega_{1, l}}\right)^{2} .
\end{aligned}
$$

And the ground state eigenfunction is

$$
\psi_{1, l}(x)=x^{\sqrt{l^{2}+M^{2} \omega_{1, l}^{2} b_{2}^{2}+\eta_{c}^{2}}} e^{-\frac{1}{2}\left[x+\frac{2 M \eta_{L}}{\Omega_{1, l}^{\frac{3}{2}}}\right] x}\left(d_{0}+d_{1} x\right) .
$$

where

$$
d_{1}=\frac{1}{\sqrt{\Omega_{1, l}}}\left[\frac{2 M \eta_{c}+\alpha \lambda B_{0} \kappa}{\left(2+\sqrt{l^{2}+M^{2} \omega_{1, l}^{2} b_{2}^{2}+\eta_{c}^{2}}\right)}+\frac{M \eta_{L}}{\Omega_{1, l}}\right] d_{0} .
$$

We can see that the lowest energy state (25) plus the ground state wavefunction (26)-(27) with the restriction on the angular frequency of the oscillator (24) is defined for the radial mode $n=1$. The presence of the Cornelltype scalar potential $S(r)$, the generalized function $f(r)$ and the Lorentz symmetry breaking parameters $\left(\alpha, B_{0}, \lambda, \kappa\right)$ modified the energy spectrum and the wave-function.

\section{Conclusions}

We have analysed the behaviour of a generalized KG-oscillator by choosing the function $f(r)$ in the equation under the effects of a central potential induced by Lorentz symmetry violation. We have chosen the function $f(r)=b_{1} r+\frac{b_{2}}{r}$ and derived the radial wave-equation in the presence of a Cornell-type scalar potential introduce by modifying the mass term 
$M \rightarrow M+S(r)$ in the equation. For a suitable wave-function, we have obtained the Heun's biconfluent differential equation and finally truncating the power series solution, the non-compact expression of the energy eigenvalues (21), and the radial wave-function (22) is obtained. By imposing the truncation condition $d_{n+1}=0$ on the eigenvalue problem, one can obtained the individual energy level and the wave-function one by one. As for example, we have obtained the energy level Eq. (25), and the radial wave-function Eqs. (26)-(27) along with the condition (23) imposed on the angular frequency of the oscillator for the lowest state of the quantum system defined by the radial mode $n=1$. Noted that for $b_{2} \rightarrow 0$, and $\eta_{c} \rightarrow 0$, the energy eigenvalues (21) reduces to the result in Ref. [38] (see Eq. (32) in the Ref. [38]). Thus the result presented in this work in comparison to those in Ref. [38] get modified due to the presence of an extra Coulomb-type potential form $\frac{\eta_{c}}{r}$ in the scalar potential $S(r)$, and the generalized function $f(r)$ where an additional term $\frac{b_{2}}{r}$ is present. Hence, the result presented in this work is more suitable than those result obtained in Ref. [38]. We see that there is a restriction (24) on the angular frequency of the oscillator $\omega_{1, l}$ for the radial mode $n=1$ that depends on the Lorentz symmetry breaking parameters $\left(\alpha, B_{0}, \lambda, \kappa\right)$ and the potential parameters which gives us a first degree polynomial solution to the Heun function $H(x)$.

\section{References}

[1] F. Ahmed, Gen. Rel. Grav. (2019) 51 : 69.

[2] F. Ahmed, Adv. High Energy Phys. 2020, 8107025 (2020).

[3] F. Ahmed, Eur. Phys. J. C 80, 211 (2020).

[4] L.-F. Deng, C.-Y. Long, Z.-W. Long and T. Xu, Adv. High Energy Phys. 2018, 2741694 (2018). 
[5] S. Zare, H. Hassanabadi and M. de Montigny, Gen. Relativ. Grav. 52, $25(2020)$.

[6] F. Ahmed, Sci. Rep. 11, 1742 (2021).

[7] F. Ahmed, EPL 130, 40003 (2020).

[8] D. Colladay and V. A. Kostelecky, Phys. Rev. D 55, 6760 (1997).

[9] D. Colladay and V.A. Kostelecky, Phys. Rev. D 58, 116002 (1998).

[10] H. Belich, T. Costa-Soares, M. M. Ferreira Jr., J. A. Helayel-Neto, F. M. O. Moucherek, Phys. Rev. D 74, 065009 (2006).

[11] G. Gazzola, H. G. Fargnoli, A. P. Ba^eta Scarpelli, M. Sampaio, M. C. Nemes, J. Phys. G : Nucl. Part. Phys. 39, 035002 (2012).

[12] H. Belich, L. P. Collato, T. Costa-Soares, J. A. Helayl-Neto, M. T. D. Orlando, Eur. Phys. J. C 62, 425 (2009).

[13] R. Casana, M. M. Ferreira Jr., V. E. Mouchrek-Santos, E. O. Silva, Phys. Lett. B 746, 171 (2015).

[14] R. Casana, C. F. Farias, M. M. Ferreira, Phys. Rev. D 92, 125024 (2015).

[15] R. Casana, M. M. Ferreira Jr., F. E. P. dos Santos, Phys. Rev. D 90, 105025 (2014).

[16] R. Casana, M. M. Ferreira Jr., E. da Hora, A. B. F. Neves, Eur. Phys. J. C 74, 3064 (2014).

[17] R. Casana, M. M. Ferreira Jr., R. V. Maluf, F. E. P. dos Santos, Phys. Lett. B 726, 815 (2013).

[18] H. Belich, T. Costa-Soares, M.M. Ferreira Jr., J.A. Helayl-Neto, Eur. Phys. J. C 41, 421 (2005). 
[19] M. A. Ajaib, Int. J. Mod. Phys. A 27, 1250139 (2012).

[20] A. G. Grushin, Phys. Rev. D 86, 045001 (2012).

[21] H. Belich, F. J. L. Leal, H. L. C. Louzada, M. T. D. Orlando, Phys. Rev. D 86, 125037 (2012).

[22] R. Casana, M. M. Ferreira Jr., E. Passos, F. E. P. dos Santos, E. O. Silva, Phys. Rev. D 87, 047701 (2013).

[23] H. Belich, L. P. Colatto, T. Costa-Soares, J. A. Helayel-Neto and M. T. D. Orlando, Eur. Phys. J. C 62, 425 (2009).

[24] H. Belich, E. O. Silva, M. M. Ferreira Jr. and M. T. D. Orlando, Phys. Rev. D 83, 12502512 (2011).

[25] L. R. Ribeiro, E. Passos, C. Furtado and J. R. Nascimento, Int. J. Mod. Phys. A 30, 1550072 (2015).

[26] K. Bakke and H. Belich, J. Phys. G: Nucl. Part. Phys. 40, 065002 (2013).

[27] K. Bakke and H. Belich, J. Phys. G: Nucl. Part. Phys. 39, 085001 (2012).

[28] L. R. Ribeiro, E. Passos and C. Furtado, J. Phys. G: Nucl. Part. Phys. 39, 105004 (2012).

[29] Q. G. Bailey and V. A. Kostelecky, Phys. Rev. D 74, 045001 (2006).

[30] V. A. Kostelecky and J. D. Tasson, Phys. Rev. Lett. 102, 010402 (2009).

[31] V. A. Kostelecky, Phys. Rev. D 69, 105009 (2004).

[32] S. Carroll, G. Field, R. Jackiw, Phys. Rev. D 41, 1231 (1990).

[33] A. P. Baeta Scarpelli, H. Belich, J. L. Boldo, L. P. Colatto, J. A. HelayelNeto, A. L. M. A. Nogueira, Nucl. Phys. Proc. Suppl. 127, 105 (2004). 
[34] V. A. Kostelecky and M. Mewes, Phys. Rev. Lett. 87, 251304 (2001).

[35] H. Belich, F. J. L. Leal, H. L. C. Louzada, M. T. D. Orlando, Phys. Rev. D 86, 125037 (2012).

[36] V. A. Kostelecky and M. Mewes, Phys. Rev. D 66, 056005 (2002).

[37] K. Bakke and H. Belich, Ann. Phys. (N. Y.) 360, 596 (2015).

[38] R. L. L. Vitoria, H. Belich and K. Bakke, Eur. Phys. J. Plus (2017) 132 : 25 .

[39] K. Bakke and H. Belich, Ann. Phys. (N. Y.) 373, 115 (2016).

[40] E. R. Figueiredo Medeiros and E. R. Bezerra de Mello, Eur. Phys. J. C (2012) $72: 2051$.

[41] M. K. Bahar and F. Yasuk, Adv. High Energy Phys. 2013, 814985 (2013).

[42] C. Alexandrou, P. de Forcrand and O. Jahn, Nuclear Phys. B (Proc. Supp.) 119, 667 (2003).

[43] A. Ronveaux, Heun's Differential Equations, Oxford University Press, Oxford ( 1995).

[44] S. Y. Slavyanov and W. Lay, Special Functions: A Unified Theory Based in Singularities, Oxford University Press, New York (2000).

[45] G. B. Arfken and H. J. Weber, Mathematical Methods For Physicists, Elsevier Academic Press, London (2005). 